\title{
Tuning dissociation using isoelectronically doped graphene and hexagonal boron nitride: Water and other small molecules
}

Yasmine S. Al-Hamdani, Dario Alfè, O. Anatole von Lilienfeld, and Angelos Michaelides'

Citation: The Journal of Chemical Physics 144, 154706 (2016); doi: 10.1063/1.4945783

View online: http://dx.doi.org/10.1063/1.4945783

View Table of Contents: http://aip.scitation.org/toc/jcp/144/15

Published by the American Institute of Physics

\section{Articles you may be interested in}

Communication: Water on hexagonal boron nitride from diffusion Monte Carlo

The Journal of Chemical Physics 142, 181101181101 (2015); 10.1063/1.4921106

Water on BN doped benzene: A hard test for exchange-correlation functionals and the impact of exact exchange on weak binding

The Journal of Chemical Physics 141, 18C53018C530 (2014); 10.1063/1.4898356

Communication: Understanding molecular representations in machine learning: The role of uniqueness and target similarity

The Journal of Chemical Physics 145, 161102161102 (2016); 10.1063/1.4964627

Electronic spectra from TDDFT and machine learning in chemical space

The Journal of Chemical Physics 143, 084111084111 (2015); 10.1063/1.4928757

Hexagonal boron nitride and water interaction parameters

The Journal of Chemical Physics 144, 164118164118 (2016); 10.1063/1.4947094

Fast and accurate predictions of covalent bonds in chemical space

The Journal of Chemical Physics 144, 174110174110 (2016); 10.1063/1.4947217

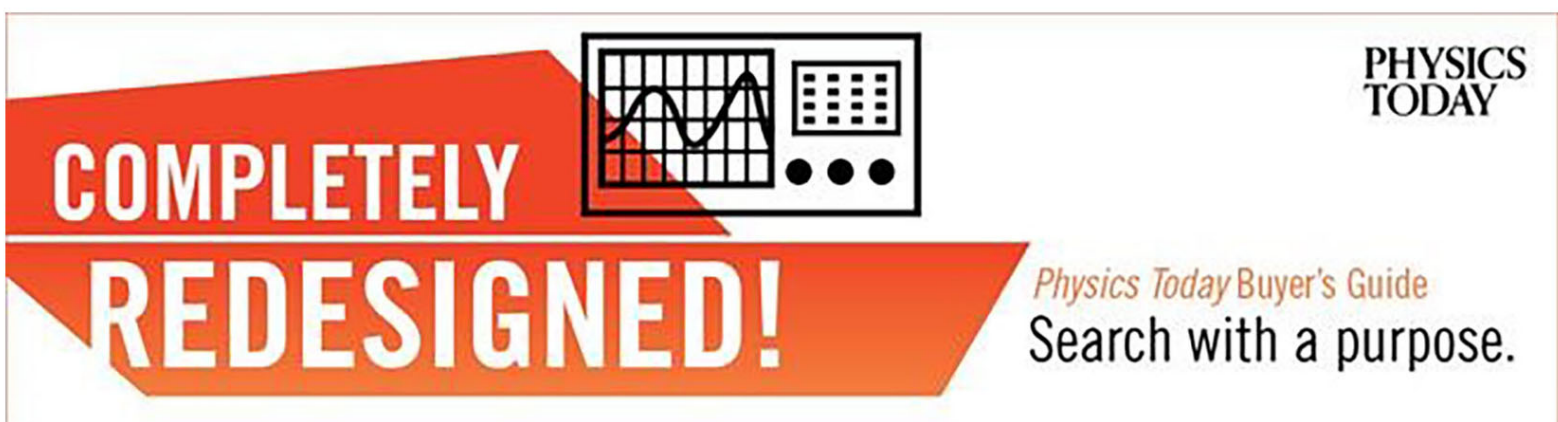




\title{
Tuning dissociation using isoelectronically doped graphene and hexagonal boron nitride: Water and other small molecules
}

\author{
Yasmine S. Al-Hamdani, ${ }^{1,2}$ Dario Alfè, ${ }^{1,3}$ O. Anatole von Lilienfeld, ${ }^{4}$ \\ and Angelos Michaelides ${ }^{1,5, a)}$ \\ ${ }^{1}$ Thomas Young Centre and London Centre for Nanotechnology, 17-19 Gordon Street, London WC1H 0AH, \\ United Kingdom \\ ${ }^{2}$ Department of Chemistry, University College London, 20 Gordon Street, London WC1H OAJ, United Kingdom \\ ${ }^{3}$ Department of Earth Sciences, University College London, Gower Street, London WC1E 6BT, United Kingdom \\ ${ }^{4}$ Institute of Physical Chemistry and National Center for Computational Design and Discovery of Novel \\ Materials (MARVEL), Department of Chemistry, University of Basel, Klingelbergstrasse 80, CH-4056 Basel, \\ Switzerland \\ ${ }^{5}$ Department of Physics and Astronomy, University College London, Gower Street, London WC1E 6BT, \\ United Kingdom
}

(Received 23 December 2015; accepted 23 March 2016; published online 21 April 2016)

\begin{abstract}
Novel uses for 2-dimensional materials like graphene and hexagonal boron nitride (h-BN) are being frequently discovered especially for membrane and catalysis applications. Still however, a great deal remains to be understood about the interaction of environmentally and industrially relevant molecules such as water with these materials. Taking inspiration from advances in hybridising graphene and h-BN, we explore using density functional theory, the dissociation of water, hydrogen, methane, and methanol on graphene, h-BN, and their isoelectronic doped counterparts: BN doped graphene and $\mathrm{C}$ doped h-BN. We find that doped surfaces are considerably more reactive than their pristine counterparts and by comparing the reactivity of several small molecules, we develop a general framework for dissociative adsorption. From this a particularly attractive consequence of isoelectronic doping emerges: substrates can be doped to enhance their reactivity specifically towards either polar or non-polar adsorbates. As such, these substrates are potentially viable candidates for selective catalysts and membranes, with the implication that a range of tuneable materials can be designed. Published by AIP Publishing. [http://dx.doi.org/10.1063/1.4945783]
\end{abstract}

\section{INTRODUCTION}

Amongst the many materials being studied for chemical applications, 2-dimensional (2D) materials like graphene and hexagonal boron nitride (h-BN) are some of the most versatile and interesting, thanks to their novel properties and sustainable compositions. Properties of graphene and h-BN manifest themselves in various important applications such as desalination, ${ }^{1}$ water purification, ${ }^{2}$ energy storage, ${ }^{3}$ energy generation, ${ }^{4-8}$ and catalysis. ${ }^{9-11}$ For example, sizeable voltages have been measured from forming water salinity gradients across graphene sheets and nanotubes, ${ }^{4-7}$ and Siria et al. demonstrated that water flowing osmotically through a BN nanotube produces remarkably large electric currents. ${ }^{8}$ This was attributed to the possible dissociation and adsorption of water on the interior of the nanotube which influences the dynamics inside the nanotube.

Much of the work on graphene and h-BN is also motivated by the sustainability and the availability of the component elements - an aspect which can be difficult to meet using materials that contain transition or noble metals. ${ }^{12}$ Already, hydrogenated h-BN is thought to be a potential photocatalyst as a material that is active under visible light and has a band gap roughly in line with the reduction and oxidation potentials

\footnotetext{
${ }^{a)}$ Electronic mail: angelos.michaelides@ucl.ac.uk
}

of water. ${ }^{9}$ Similar efforts are being made to develop graphene into a photocatalyst by modification of its band gap, and also as a support to other photocatalytic materials. ${ }^{10,11}$

Despite the promising applications of h-BN and graphene as membranes and catalysts, there are still major gaps in our understanding of the interaction of molecules like water on clean graphene and h-BN surfaces on the atomic level, and even less is known about how doping in the materials alters their interaction with molecules. Indeed, experimental routes to produce hybrid composites of h-BN and graphene $\mathrm{e}^{13,14}$ have emerged with high levels of control being reported on the nanometre scale, which is another reason to gain better atomic level understanding. Various theoretical studies on band gap engineering using $\mathrm{h}-\mathrm{BN}$ and graphene mixtures, ${ }^{15-23}$ have revealed the tuneability of these materials through the mixture of atoms. Other studies have focused on exploiting this tuneability for catalysis of oxygen reduction reactions, ${ }^{24-33}$ water transport, ${ }^{34}$ and $\mathrm{H}_{2}$ adsorption. ${ }^{35-37}$

An important aspect to consider, if using graphene and $\mathrm{h}-\mathrm{BN}$ based materials as catalysts, is their degree of selectivity. A high degree of selectivity is an extremely desirable property for any catalyst and indeed, the rational design of metalbased heterogeneous catalysts is the focus of intense research (see, e.g., Refs. 38-44). However, even in these cases, the metal-based catalysts do not necessarily have very different selectivities, and although they can be doped or alloyed 
to vary their reactivity, the effect on reaction energies and barriers is often a constant shift with respect to different molecules. ${ }^{44-47}$ For instance, in the reaction pathways towards $\mathrm{H}_{2}$ formation discussed by Cortright et al., a metal catalyst is used throughout, which also catalyses $\mathrm{H}_{2}$ consuming reactions instead. ${ }^{48}$ Meanwhile, Guo et al. have shown that a more complex selective catalyst gives rise to a higher conversion rate of methane to $\mathrm{H}_{2}{ }^{49}$

Here we investigate water and some other environmentally and industrially relevant small molecules with density functional theory (DFT). The particular focus of this study is to establish the thermodynamics of dissociative adsorption and how this is affected by doping. From this work we draw a number of conclusions. First, doping strongly affects the dissociation process, in some situations making dissociation more favourable by several electronvolts. Second, different surfaces have varying reactivity for the set of molecules considered, with some substrates significantly enhancing the reactivity of polar molecules and others enhancing the reactivity of non-polar adsorbates.

Below, we begin by describing our computational setup in Section II and present our DFT results for water adsorption in Section III A, followed by an overview regarding the relative adsorption of other molecules in Section III B. In Section IV we discuss the trends observed in adsorption sites and structures, and propose a general framework for dissociative adsorption before finally concluding, in Section V.

\section{METHODS}

The dissociative adsorption of a water monomer and other molecules on graphene, h-BN, and their doped counterparts was calculated using DFT and the Vienna $A b$ Initio Simulation Package (VASP) 5.3.2. ${ }^{50-53}$ VASP uses plane-wave basis sets and projector augmented wave (PAW) potentials ${ }^{54,55}$ to model the core region of atoms.

\section{A. System setup}

The graphene and h-BN substrates are modelled using $(5 \times 5)$ hexagonal unit cells containing 50 atoms, for which adsorption energies are converged to less than $10 \mathrm{meV}$ with respect to $(7 \times 7)$ unit cells. After a series of convergence tests for the plane-wave cutoff energy, we chose to use a $400 \mathrm{eV}$ energy cutoff, which gives dissociative adsorption energies converged to within $16 \mathrm{meV}$ of a $600 \mathrm{eV}$ energy cutoff. $\Gamma$-point sampling of reciprocal space for the $(5 \times 5)$ cell was used but $\mathbf{k}$-point densities up to $(9 \times 9 \times 1)$ were tested. Adsorption energies using $\Gamma$-point sampling are within $50 \mathrm{meV}(3 \%)$ of the converged adsorption energies for all substrates. Spin polarisation was applied since $\mathrm{H}$ preadsorption on the substrates gives rise to spin polarised states. A $10 \AA$ separation in the $\mathrm{z}$-direction between substrates without a dipole correction proved to be converged for dissociative adsorption energies of water compared to using a dipole correction or a $20 \AA$ separation ( $<15 \mathrm{meV}$ difference $).{ }^{56}$

For the dissociative adsorption energies evaluated here (spanning a few eV) we have mostly used the PerdewBurke-Ernzerhof $(\mathrm{PBE})^{57}$ generalised gradient approximation exchange-correlation functional. However we have also verified that the key results obtained here are not particularly sensitive to the choice of exchange-correlation functional, as discussed in Section IV.

There are many different ways of isoelectronically doping graphene with $\mathrm{BN}$ and vice versa and as a first step we focus on low concentrations of doping: one pair of BN substituting two $C$ atoms in a $(5 \times 5)$ unit cell of graphene which we refer to as boron nitride doped graphene (BNDG) and likewise, two $\mathrm{C}$ atoms substituting a $\mathrm{BN}$ pair in a $(5 \times 5)$ unit cell of h-BN, henceforth referred to as $2 \mathrm{CBN}$ (Fig. 1). Doped substrates are modelled by isoelectronically doping the pristine sheets and relaxing the unit cells using a plane-wave energy cutoff of $600 \mathrm{eV}$ to remove any strain introduced by the mixture of B, $\mathrm{N}$ and $\mathrm{C}$ atoms. Relaxation effects are small: less than $1 \%$ of the relaxed lattice constant of the undoped system. ${ }^{58}$

When water dissociates on a 2D substrate there are a number of possible adsorption scenarios. Here, we have focused on four possible outcomes. Schematic illustrations are given in Fig. 2 and in brief they involve the following: (i) An $\mathrm{OH}$ group on the surface and the release of (half) an $\mathrm{H}_{2}$ molecule, referred to as "OH $\left(1 / 2 \mathrm{H}_{2}\right.$ gas)"; (ii) the adsorption of both $\mathrm{OH}$ and $\mathrm{H}$ components of water on the surface, with them both being on one side of the substrate, namely, "cis $(\mathrm{OH}-\mathrm{H})$." We consider this configuration to be particularly important because 2D materials tend to be examined by supporting them on other materials, leaving only one side of the surface exposed; (iii) the adsorption of both $\mathrm{OH}$ and $\mathrm{H}$ on the surface but this time on opposite sides of the substrate, referred to here as "trans $(\mathrm{OH}-\mathrm{H})$ ". This could arise from having the substrate suspended in a wet environment or from the $\mathrm{H}$ atoms diffusing through the sheet and there are indications that graphene and h-BN are permeable to protons. ${ }^{59}$ However, since it is not clear how likely it is for molecules to dissociate on different sides of the substrates, we consider this $\operatorname{trans}(\mathrm{OH}-\mathrm{H})$ configuration to be less relevant

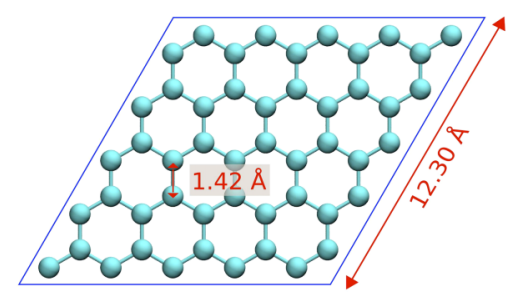

(a) pristine graphene

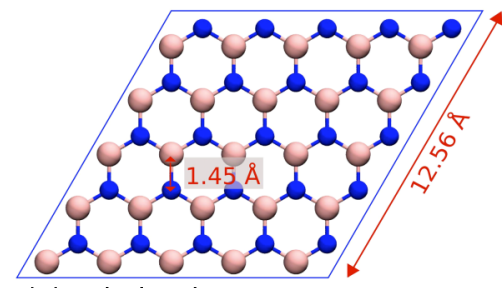

(c) pristine h-BN

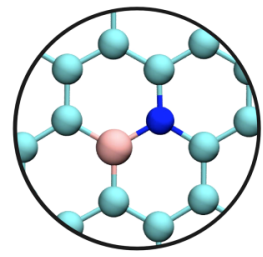

(b) BNDG

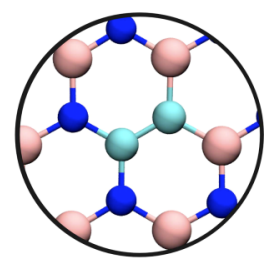

(d) $2 \mathrm{CBN}$
FIG. 1. The clean and doped graphene and h-BN surfaces considered in this study. (a) $(5 \times 5)$ unit cell of graphene. (b) $\mathrm{BN}$ doping in $(5 \times 5)$ unit cell of graphene which we refer to as BNDG. (c) $(5 \times 5)$ unit cell of h-BN. (d) C doping in $(5 \times 5)$ unit cell of h-BN, referred to as $2 \mathrm{CBN}$. For clarity only a small portion of the $(5 \times 5)$ unit cell is shown in (b) and (d). $C$ is coloured cyan, $\mathrm{B}$ is pink, and $\mathrm{N}$ is blue. 

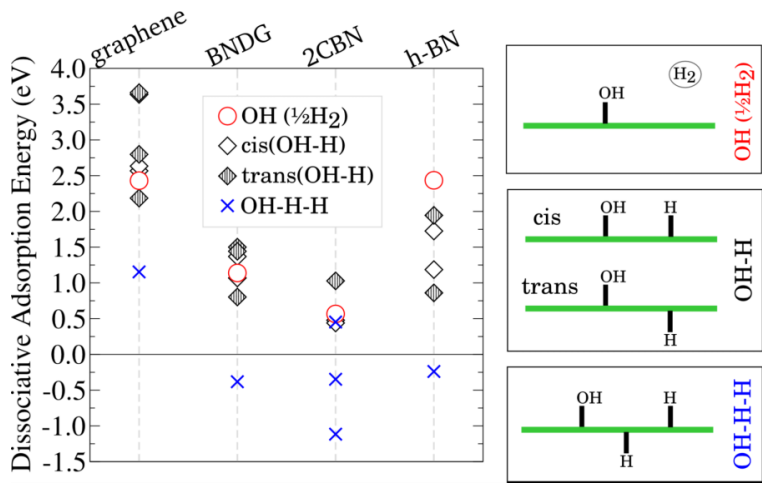

FIG. 2. The dissociative adsorption energy of water on graphene, BNDG, $2 \mathrm{CBN}$, and h-BN is shown for different adsorption structures. Red circles indicate the adsorption of $\mathrm{OH}$ from water onto the substrate and the release of hydrogen gas. The black diamonds indicate the dissociative adsorption of a water molecule into $\mathrm{OH}$ and $\mathrm{H}$ on the substrate. The blue crosses correspond to the adsorption energies on a hydrogenated substrate. The categories of water dissociation on the substrate are illustrated on the right.

than $\operatorname{cis}(\mathrm{OH}-\mathrm{H})$; (iv) lastly, "OH-H-H" which is again the adsorption of both $\mathrm{OH}$ and $\mathrm{H}$, this time on a surface that has an $\mathrm{H}$ atom pre-adsorbed. We tested this particular setup in light of previous experimental and simulation work, where this is thought to cause water dissociation. ${ }^{8}$ Many adsorption sites are available for each category and we have calculated only a number of possibilities: ortho, meta, and para-positioning of the adsorbed components with respect to each other, as well as adsorption of the components far away from each other and the doping site in the substrate.

The absolute adsorption energy for dissociative adsorption, $E_{a d s}$ is defined as

$$
E_{a d s}=E_{a d s / s u b}^{t o t}-E_{s u b}^{t o t}-E_{a d s}^{t o t},
$$

where $E_{\text {ads } / \text { sub }}^{\text {tot }}$ is the total energy of the adsorption system, $E_{s u b}^{\text {tot }}$ is the total energy of the relaxed substrate, and $E_{a d s}^{\text {tot }}$ is the energy of the intact molecule in the gas phase. Equation (1) is used for all but one adsorption state, that is $\mathrm{OH}\left(1 / 2 \mathrm{H}_{2}\right.$ gas). Here we also take into account the energy $\left(E_{\mathrm{H}_{2}}^{\text {tot }}\right)$ of the $1 / 2 \mathrm{H}_{2}$ gas molecule that is formed,

$$
E_{a d s}=E_{a d s / s u b}^{t o t}+1 / 2 E_{H_{2}}^{t o t}-E_{s u b}^{t o t}-E_{a d s}^{t o t} .
$$

Within these definitions, negative adsorption energies correspond to favourable (exothermic) adsorption processes. Bond strengths of hydrogen and hydroxyl to the surfaces are calculated with respect to a gas phase hydrogen atom or hydroxyl group instead of the whole molecule,

$$
E_{\text {bond }}=E_{\text {sub }}^{\text {tot }}+E_{\text {ads }}^{\text {tot }}-E_{\text {ads } / \text { sub }}^{\text {tot }} \text {. }
$$

\section{RESULTS}

We begin with the results for the dissociative adsorption of water on the pure substrates, graphene and h-BN, and on the doped substrates, BNDG and 2CBN. In general, we find that the dissociation of water is more facile on the doped substrates and is also strongly affected by the presence of a pre-adsorbed $\mathrm{H}$ atom, local electronic induction, and steric effects arising from rehybridisation of orbitals in the substrate atoms. We use these insights to look at the adsorption of $\mathrm{H}_{2}$, methane, and methanol on the same surfaces in Section III B. From our analysis, we see that different substrates favour the dissociation of different molecules, depending on their polarity, enabling us to make comparisons between the adsorption behaviour of polar and non-polar molecules and fragments.

\section{A. Dissociative adsorption of water on graphene, h-BN, BNDG, and 2CBN}

Fig. 2 reports results for the dissociative adsorption of water on the clean and doped substrates. It can be seen that the energetics of the dissociation process varies significantly for the various adsorption structures and substrates.

On pristine graphene we find that dissociation is strongly endothermic in agreement with previous work. ${ }^{60,61}$ In addition the energy of the dissociation process varies by as much as $2 \mathrm{eV}$ depending on the final adsorption configuration. The lowest adsorption configuration on pristine graphene is $\operatorname{trans}(\mathrm{OH}-\mathrm{H})(2.19 \mathrm{eV})$ with $\mathrm{OH}$ and $\mathrm{H}$ in ortho positions, in agreement with the work of $\mathrm{Xu}$ et al. ${ }^{61} \mathrm{The} \operatorname{cis}(\mathrm{OH}-\mathrm{H})$ configuration shown in Fig. 3(a) on graphene has a dissociative adsorption energy of $2.57 \mathrm{eV}$ and is thus $\sim 0.4 \mathrm{eV}$ less stable than $\operatorname{trans}(\mathrm{OH}-\mathrm{H})$. Dissociative water adsorption is in general more thermodynamically favourable on h-BN than on graphene. For example, the cis $(\mathrm{OH}-\mathrm{H})$ state on pristine h-BN shown in Fig. 3(d) has $E_{a d s}$ of $1.19 \mathrm{eV}$ and is $1.38 \mathrm{eV}$ more favourable than the equivalent structure on graphene. Nonetheless, given just how thermodynamically unfavourable water dissociation is, it is unlikely that water monomers will dissociate on pristine graphene and $\mathrm{h}-\mathrm{BN}$.

Upon moving to the doped substrates, for which numerous configurations were considered, we find a significant lowering in the energy to adsorb water. From graphene to BNDG, and from h-BN to $2 \mathrm{CBN}$, we gain $\sim 1 \mathrm{eV}$ in the adsorption of a water molecule. The $\operatorname{cis}(\mathrm{OH}-\mathrm{H})$ state and lowest energy dissociation state for each doped surface is shown in Fig. 3. On both BNDG and $2 \mathrm{CBN}, \mathrm{B}-\mathrm{OH}$ and $\mathrm{C}-\mathrm{H}$ bonds are formed. Note from Table I that the $\mathrm{B}-\mathrm{OH}$ bond is $\sim 1.3 \mathrm{eV}$ stronger on BNDG than on h-BN (or $\mathrm{C}-\mathrm{OH}$ on graphene). Hence, a marked activation of the $\mathrm{B}$ atom towards binding $\mathrm{OH}$ results from the mixture of $\mathrm{N}$ and $\mathrm{C}$ atoms surrounding it and in this

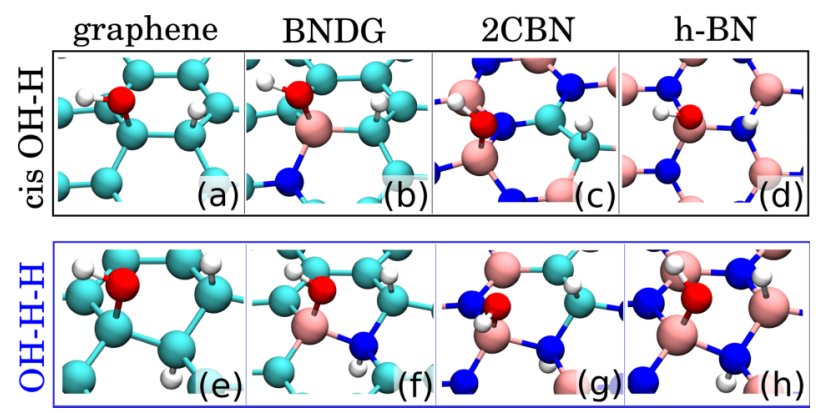

FIG. 3. The most stable $c i s(\mathrm{OH}-\mathrm{H})$ (top panel) and most stable overall dissociative adsorption structures (lower panel) of water on graphene, h-BN, BNDG and $2 \mathrm{CBN}$ are shown. (a) and (e) are water on pristine and hydrogenated graphene, respectively. (b) and (c) show water adsorbed on BNDG and $2 \mathrm{CBN}$, whilst (f) and (g) show water adsorption on the hydrogenated counterparts. (d) and (h) are on pristine and hydrogenated h-BN, respectively. 
TABLE I. Bond strengths (in eV) for $\mathrm{H}$ and $\mathrm{OH}$ on graphene, h-BN, and BNDG sheets with respect to a gas phase hydrogen atom or $\mathrm{OH}$ molecule. Parentheses indicate neighbouring atoms in the substrate. Negative bond energies correspond to endothermic but metastable adsorption minima. No minimum was found for $\mathrm{OH}$ adsorbed on the $\mathrm{N}$ atom.

\begin{tabular}{lc}
\hline \hline Bond & Bond strength (eV) \\
\hline Graphene & 0.81 \\
C-H & 0.67 \\
C-OH & \\
\hline h-BN & -0.77 \\
N-H & -0.01 \\
B-H & 0.67 \\
B-OH & \\
BNDG & 0.98 \\
B-H & 0.07 \\
N-H & 1.15 \\
(B)C-H & 1.04 \\
(N)C-H & 1.96 \\
B-OH & 0.84 \\
(B)C-OH & 1.03 \\
(N)C-OH & \\
\hline \hline
\end{tabular}

way doping leads to a considerable lowering of the dissociative adsorption energy.

The presence of the pre-adsorbed $\mathrm{H}$ atom also significantly improves the thermodynamics of water adsorption by $\sim 1 \mathrm{eV}$ for each substrate. Favourable $\mathrm{OH}-\mathrm{H}-\mathrm{H}$ configurations are shown in Fig. 3, and from Fig. 2 it can be seen that water splitting is thermodynamically favourable on the hydrogenated h-BN $(-0.24 \mathrm{eV})$, BNDG $(-0.38 \mathrm{eV})$, and 2CBN $(-1.12 \mathrm{eV})$ surfaces. Thus doping and hydrogenating both graphene and h-BN makes the thermodynamics of water dissociation considerably more favourable. The general conclusion that pre-adsorbed hydrogen facilitates water dissociation is in agreement with the work of Siria et $a l{ }^{8}$ Interestingly, the overall most favourable states for water dissociation on the doped surfaces contain a $\mathrm{B}-\mathrm{N}-\mathrm{C}$ construction in the surface where $\mathrm{B}-\mathrm{OH}, \mathrm{N}-\mathrm{H}$, and $\mathrm{C}-\mathrm{H}$ bonds are formed. We considered if the increased reactivity at these sites is due to the pre-adsorbed $\mathrm{H}$ atom on a $\mathrm{N}$ site destabilising the surface and thus activating it towards water adsorption, but this is unlikely because the $\mathrm{N}-\mathrm{H}$ bond is very weak (only $0.07 \mathrm{eV}$ ). The B-N-C construction in the surface of doped substrates is therefore central to making the dissociation energy more exothermic and exemplifies the use of isoelectronic doping to tune the dissociative adsorption energy of water. In all $\mathrm{OH}-\mathrm{H}-\mathrm{H}$ states, the $\mathrm{OH}$ and $\mathrm{H}$ components of the dissociated water are arranged in a hydrogen bonded fashion. The hydrogen bond on h-BN at $1.95 \AA$ is shorter than the hydrogen bond on graphene $(2.23 \AA$ ) despite the slightly smaller lattice constant of graphene. The hydrogen bonding distances are indicative of the more polarised binding of $\mathrm{OH}$ and $\mathrm{H}$ on $\mathrm{h}-\mathrm{BN}$, which culminates in a more negative oxygen atom in the $\mathrm{OH}$ group and hence a shorter hydrogen bond.

Additional DFT calculations of water dissociation on the protonated (as opposed to hydrogenated) substrates were also performed. A homogeneous background charge is added in the DFT calculations of the charged systems so that the electrostatic interactions do not diverge and can be computed under periodic boundary conditions. These reveal that protonation is slightly less effective than hydrogenation but still increases the tendency of water to dissociate by $\sim 0.8 \mathrm{eV}$ with respect to the non-protonated clean surfaces. Thus either hydrogen pre-adsorption or acidic conditions (preadsorbed protons) could be key elements in the activation of these sheets towards dissociative water adsorption.

Before moving on to discuss the other adsorbates, two additional features of these adsorption systems deserve comment. First, adsorption of the dissociated fragments on separate sides of the sheet (so-called trans-adsorption) is favoured in general. Specifically, trans-ortho $(\mathrm{OH}-\mathrm{H})$ adsorption is $\sim 0.4 \mathrm{eV}$ more stable than cis-ortho $(\mathrm{OH}-\mathrm{H})$ on graphene. This is consistent with previous work on graphene ${ }^{62-66}$ and demonstrates the stabilisation gained by adhering to a more tetrahedral structure around the $\mathrm{sp}^{3}$ hybridised $\mathrm{C}$ atom. Likewise on h-BN and BNDG, the tetrahedral arrangements of $\operatorname{trans}(\mathrm{OH}-\mathrm{H})$ and $\mathrm{OH}-\mathrm{H}-\mathrm{H}$ lead to lower dissociative adsorption energies (by about $0.3 \mathrm{eV}$ ). Note the $2 \mathrm{CBN}$ system is an exception and the most stable $(\mathrm{OH}-\mathrm{H})$ configuration on $2 \mathrm{CBN}$ has cis-para-positioning, shown in Fig. 3(c). The trans-ortho $(\mathrm{OH}-\mathrm{H})$ state on $2 \mathrm{CBN}$ is still close in energy and only $0.04 \mathrm{eV}$ less stable than cis-para. ${ }^{67}$ This can be explained by the difference in partial charges on the $\mathrm{B}$ atoms bonding to $\mathrm{OH}$ in each case. Electronegative $\mathrm{N}$ atom neighbours make $\mathrm{B}$ atoms more positive and subsequently form a stronger polar bond with $\mathrm{OH}$. In the trans-ortho state, the B atom is surrounded by only two $\mathrm{N}$ atoms and hence is not as electrophilic as the $\mathrm{B}$ atom in the cis-para state which is bonded to three other $\mathrm{N}$ atoms. This example in $2 \mathrm{CBN}$ demonstrates that inductive effects from neighbouring atoms dominate over steric effects. Despite the advantage of satisfying the $\mathrm{sp}^{3}$ hybridisation in trans-adsorption states, it is important to remember that in practice $2 \mathrm{D}$ materials are often suspended or grown over substrates ${ }^{68-76}$ (metals or silicon carbide) where cis configurations are more likely.

Second, inductive effects are also introduced by the adsorbed water fragments. This can be seen by comparing the co-adsorbed to the separately adsorbed $\mathrm{OH}$ and $\mathrm{H}$ fragments. Specifically, $\mathrm{OH}\left(1 / 2 \mathrm{H}_{2}\right.$ gas) adsorption on graphene and h-BN only differ by $5 \mathrm{meV}$ and indeed the $\mathrm{C}-\mathrm{OH}$ and $\mathrm{B}-\mathrm{OH}$ bonds (as listed in Table I) in graphene and h-BN are almost identical. In contrast, $\mathrm{C}-\mathrm{H}$ bonds in graphene are significantly stronger than $\mathrm{N}-\mathrm{H}$ bonds in h-BN, implying that $\mathrm{OH}-\mathrm{H}$ on graphene might be more stable, and yet water adsorption is more exothermic on h-BN. It follows that the binding of hydrogen atoms on the surface perturbs the local electronic structure and therefore, the bond strength of $\mathrm{OH}$ to the surface, such that the $\mathrm{OH}-\mathrm{H}$ configuration is considerably more stable on h-BN than on graphene.

It is useful to explain these trends in terms of the physical properties of the surfaces and we have done this by looking at Bader charges, ${ }^{77,78}$ average electrostatic potentials at each atom, and Kohn-Sham orbitals of the dissociated states. ${ }^{79}$ Comparison of the adsorption structures and Bader charges suggests the most stable adsorption states arise from the 
following: (i) $\mathrm{C}-\mathrm{H}$ in which the $\mathrm{C}$ site has the most negative partial charge across the surface; (ii) $\mathrm{B}-\mathrm{OH}$ in which the $\mathrm{B}$ atom is positive and susceptible to nucleophilic attack; and (iii) $\mathrm{N}-\mathrm{H}$ in which the $\mathrm{N}$ atom is the most negative and therefore the strongest nucleophile. A careful analysis reveals that the adsorption of water is affected by a combination of factors involving orbital overlap and electrostatic interactions. Graphene has weaker electrostatic interactions with water than h-BN, but better orbital overlap (evidenced by bond strengths in Table I). In contrast, hybrids of h-BN and graphene have stronger electrostatic interactions with water than graphene, and also stronger orbital overlap with water than h-BN. Due to these combined effects, doped graphene and h-BN are more suited for the adsorption of water. Evidently for a given substrate, electrostatic interactions with a molecule are determining the site of adsorption (e.g., in 2CBN the cis-para state of water is more stable than the trans-ortho).

To recap, isoelectronic doping has a significant impact on the thermodynamics of water dissociation of graphene and h-BN. The most thermodynamically favourable adsorption identified is the $\mathrm{OH}-\mathrm{H}-\mathrm{H}$ configuration on $2 \mathrm{CBN}$ with an adsorption energy of $-1.12 \mathrm{eV}$. The strong adsorption energy on $2 \mathrm{CBN}$ can be attributed to the following: (i) the $\mathrm{B}-\mathrm{OH}$ bond in which the $\mathrm{B}$ atom is more positive compared to $\mathrm{B}$ atoms in the other substrates; and (ii) a stronger $\mathrm{C}-\mathrm{H}$ bond at $2 \mathrm{CBN}$ as opposed to a $\mathrm{B}-\mathrm{H}$ bond at h-BN.

\section{B. Dissociative adsorption of hydrogen, methane, and methanol}

With the insight gained from water adsorption, we also calculated the dissociative adsorption of $\mathrm{H}_{2}$, methane, and methanol. As before, various configurations were calculated for each system, and in Fig. 4(a) we report the most favourable dissociation energies found for the molecules on the same side (cis configurations) of the pure and doped substrates. The change in zero point energy (ZPE) upon dissociative adsorption for each system is also included in the energies in Fig. 4. ZPEs were calculated using the harmonic approximation and we find that the change in ZPE increases the dissociative adsorption energies by up to $0.3 \mathrm{eV}$, which is certainly not insignificant. In some cases, the adsorption energies of the trans-states are more favourable than cis but since it is more feasible for adsorbates to dissociate on the same side of the substrate, we show results only for cis configurations.

From these calculations with the other adsorbates, we learn two key things. First, doping of the pristine substrates helps the thermodynamics of dissociation for these molecules too. Second, the details are quite different, with methanol behaving in a similar manner to water by benefiting most from $\mathrm{BN}$ doping in graphene, whereas $\mathrm{H}_{2}$ and methane benefit most from C doping in h-BN. Figs. 4(b) and 4(c) illustrate this latter point by showing the gain in dissociative adsorption energy for each molecule as a result of doping in the pristine substrates.

The adsorption of methanol varies in a similar way to water across the different substrates and favours the same adsorption sites (ortho on graphene, BNDG, and h-BN, and
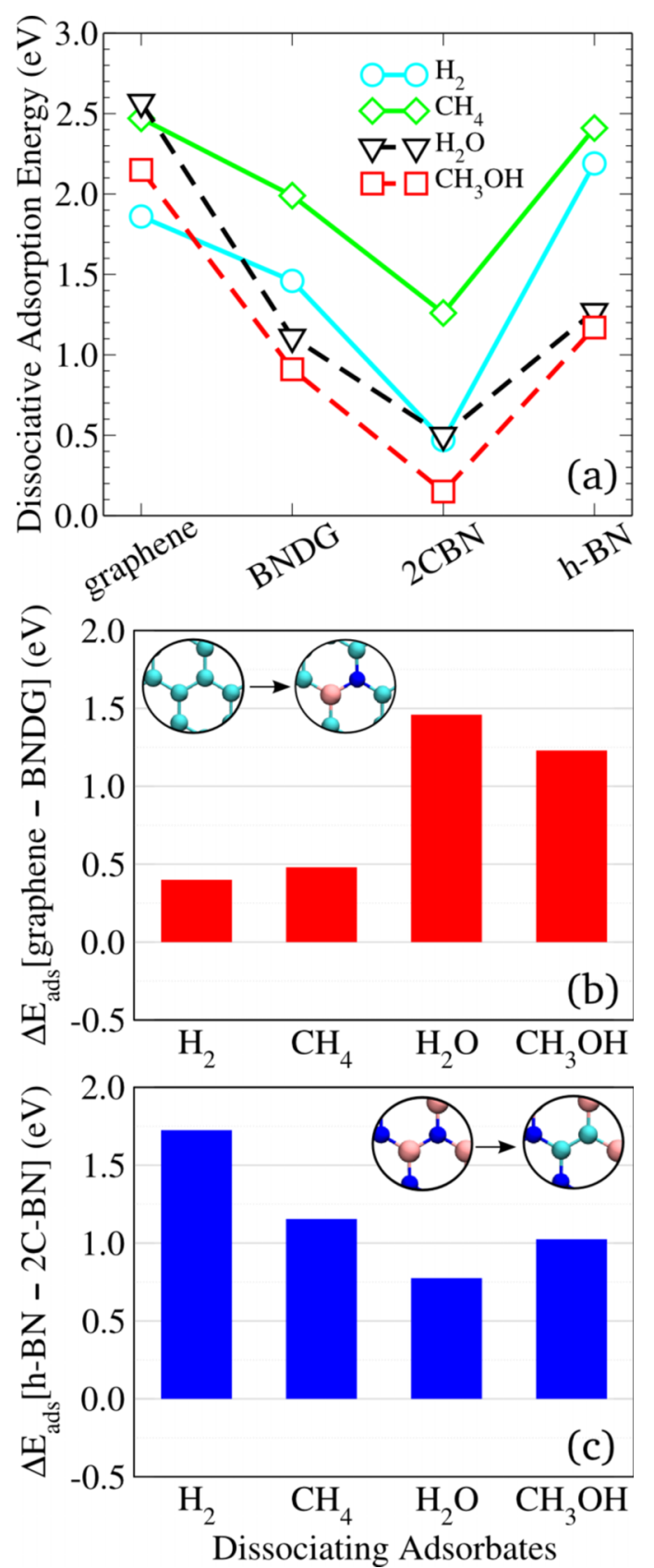

FIG. 4. (a) Dissociative adsorption energies including ZPE contributions of $\mathrm{H}_{2}$, methane, water, and methanol on graphene, BNDG, $2 \mathrm{CBN}$, and h-BN. $\mathrm{H}_{2}$ in blue circles, methane in green diamonds, water in black triangles, and methanol in red squares. Results are given only for the most stable adsorption structure for each molecule and substrate with the fragments adsorbed on the same side of the substrate and without pre-adsorbed hydrogen. (b) Gain in adsorption energies from doping pristine graphene with $\mathrm{BN}$ (in $\mathrm{eV}$ ) for different molecules, illustrating a marked increase in the reactivity with polar adsorbates. (c) Gain in adsorption energies from doping pristine h-BN with $2 \mathrm{C}$ (in $\mathrm{eV}$ ) for different molecules and here the reactivity with non-polar adsorbates increases more significantly. The insets in (b) and (c) illustrate the doping.

para on 2CBN). From Fig. 4, we see that water and methanol adsorption energies both become more favourable by $\sim 1.4 \mathrm{eV}$ as the substrate is changed from graphene to BNDG. Having established that the $\mathrm{C}-\mathrm{OH}$ to $\mathrm{B}-\mathrm{OH}$ change in bond energy is the main contributor to the difference in adsorption energies for water on graphene and BNDG, we can deduce that the 
same is true for methanol. Note that the adsorption of methanol is stronger than that of water on all substrates by $0.2-0.4 \mathrm{eV}$. On graphene, BNDG, and $2 \mathrm{CBN}$ the $\mathrm{C}-\mathrm{O}$ bond of methanol is broken preferentially with the $\mathrm{CH}_{3}$ fragment bonding to the substrate at the same sites as the $\mathrm{H}$ from water does. However on $\mathrm{h}-\mathrm{BN}$, the $\mathrm{O}-\mathrm{H}$ bond is broken instead, resulting in $\mathrm{N}-\mathrm{H}$ and $\mathrm{B}-\mathrm{OCH}_{3}$ bonds with the h-BN substrate.

Meanwhile the non-polar molecules, $\mathrm{H}_{2}$ and methane, also benefit from doping of the pristine substrates but in particular from $\mathrm{C}$ doping in h-BN. This appears to be because the alkene-like bond between the two $\mathrm{C}$ atoms, which is susceptible to alkene addition reactions, is particularly effective at breaking weakly polarised bonds. Methane and $\mathrm{H}_{2}$ follow exactly the same trend but $\mathrm{H}_{2}$ is adsorbed around $0.6 \mathrm{eV}$ more strongly overall.

By tracking the lowest adsorption states across the substrates in Fig. 4(a), we see that the preference for $\mathrm{H}_{2}$ and water switch; $\mathrm{H}_{2}$ adsorbs preferably on graphene and water is preferred on BNDG and pure h-BN. $\mathrm{H}_{2}$ and water have almost the same dissociative adsorption energies on $2 \mathrm{CBN}(\sim 0.5 \mathrm{eV})$. The different adsorption preferences that depend on the isoelectronic substrate doping is a significant outcome, especially given that these materials are composed of sustainable and abundant elements, making them desirable candidates for catalysis.

Finally, as with water adsorption, we also examined the effect of $\mathrm{H}$ pre-adsorption on the dissociative adsorption energy of these small molecules. We found in a similar manner to water that dissociative adsorption becomes more favourable by $0.7-1.5 \mathrm{eV}$ on the hydrogenated surfaces, such that $\mathrm{H}_{2}$, water, and methanol have exothermic dissociative adsorption energies on BNDG, $2 \mathrm{CBN}$, and h-BN. Thus, as with water, doping and hydrogenation significantly improve the energetics of dissociative adsorption on graphene and h-BN.

\section{DISCUSSION AND GENERAL FRAMEWORK}

Some important trends can be observed from the adsorption structures and energies of water and the other molecules studied here, which are likely to apply in general to polar and non-polar adsorbates on BNDG and $\mathrm{C}$ doped h-BN systems. Although we have studied water adsorption more extensively, the trends also hold for $\mathrm{H}_{2}$, methanol, and methane. We summarise as follows:

- Isoelectronic doping of graphene with $\mathrm{BN}$ increases the reactivity with polar adsorbates (i.e., water and methanol) by $\sim 1.4 \mathrm{eV}$ but only changes the reactivity with non-polar adsorbates by $\sim 0.5 \mathrm{eV}$. Conversely, isoelectronic doping of $\mathrm{h}-\mathrm{BN}$ with $\mathrm{C}$ increases the reactivity most with $\mathrm{H}_{2}$ and methane, by $1.2-1.8 \mathrm{eV}$.

- Hydrogen atom (or proton) pre-adsorption on the substrate significantly improves the thermodynamics of dissociation for the molecules considered by $\sim 1 \mathrm{eV}(\sim 0.8 \mathrm{eV})$, resulting in exothermic dissociative adsorption, and suggesting that acidic conditions aid dissociation on the substrates.

- The most exothermic adsorption sites for polar adsorbates share the $\mathrm{B}-\mathrm{N}-\mathrm{C}$ construction in which there is already a $\mathrm{H}$ atom pre-adsorbed on a $\mathrm{N}$ atom. Meanwhile, non-polar adsorbates favour $\mathrm{C}-\mathrm{C}$ sites with localised electrons (as in $2 \mathrm{CBN}$ ).

- Local electronic inductive effects dominate over steric effects. In other words, para-positioning of molecule fragments is possible (however ortho is generally favoured) if the atoms in the substrate have a larger electrostatic potential in the para-sites.

- Atoms in the substrate that change to $\mathrm{sp}^{3}$ hybridisation as a result of chemisorption prefer to be in a more tetrahedral arrangement, e.g., the trans-ortho configuration is $\sim 0.3 \mathrm{eV}$ more stable than cis-ortho.

Some comments related to these trends are appropriate. First, all the numbers given have been derived from the PBE exchange-correlation functional. It is well known that bond strengths and adsorption energies vary from one functional to the next ${ }^{80,81}$ and PBE in particular neglects van der Waals (vdW) dispersion forces and does not include exact exchange. Indeed, previous work on similar systems to those considered here, namely, the physisorption of water on $\mathrm{h}-\mathrm{BN}^{82}$ and on BN doped benzene, ${ }^{83}$ has shown that vdW interactions can be important. Here, however, we are concerned with strongly bonded chemisorption structures of the dissociated fragments of water and the other molecules involving an energy scale of several electronvolts. Nonetheless we have investigated the dissociative adsorption energies for all states in Fig. 4 using the vdW-inclusive optB86b-vdW functional. ${ }^{84-86}$ We find that the inclusion of vdW interactions makes the thermodynamics of dissociative adsorption energy more favourable by $0.2-0.5 \mathrm{eV}$. With this functional, some adsorption states are exothermic even in the absence of pre-adsorbed hydrogen. In contrast, when we look at the thermodynamics of water adsorption with B3LYP, ${ }^{87-90}$ that accounts for some exact exchange but not dispersion, dissociative adsorption is less favourable by circa $0.2-0.4 \mathrm{eV}$. It is clear therefore that the thermodynamics of dissociative adsorption is sensitive to the choice of exchangecorrelation functional, with the PBE values presented here resting in the middle of three functionals considered. Importantly, the relative energies and trends across the surfaces remain unchanged whether or not dispersion interactions or exact exchange are accounted for.

Second, when probed experimentally 2D materials like graphene and h-BN are often adsorbed on a support material such as metals or silicon carbide. We have not included supporting materials in this study but the electronic properties of graphene and h-BN can be influenced by the choice of support. ${ }^{68-75}$ Metals, for instance, can hybridise the $\mathrm{p}_{z}$-states in graphene and the $\mathrm{N}$ atoms in $\mathrm{h}-\mathrm{BN}$, and thus alter the reactivity of the surfaces. ${ }^{69,71,72}$ It is also known that differences in the lattice constants of the 2D material and support can lead to an undulating moiré structure in which different regions of the 2D overlayer interact differently with the substrate. . $^{2,70,73,75,76}$ It would be interesting in future work to explore how the presence of a substrate alters the trends observed here.

Third, we have seen that depending on the type of doping, the thermodynamics of dissociation of either polar or nonpolar molecules can be enhanced. This would potentially be 
exploited in heterogeneous catalysis where it is generally desirable to identify catalysts that can cleave specific bonds and as a result enhance the selectivity towards a particular reaction product. In future work, it would be interesting to explore this possibility through calculations of the kinetics of dissociation on the substrates considered here. However, since it is now well established that reaction barriers for chemical reactions at surfaces correlate well with the thermodynamics, it is not unreasonable to suggest that the thermodynamic trends identified here could lead to interesting catalytic behaviour.

\section{CONCLUSION}

To conclude, the dissociative adsorption of water, $\mathrm{H}_{2}$, methane, and methanol has been studied on pristine graphene and h-BN, and on their doped counterparts (BNDG and $2 \mathrm{CBN}$ ) using DFT. Most notably, isoelectronic doping of the pristine surfaces makes the dissociation process more favourable generally by at least $1 \mathrm{eV}$. Based on electronic structure analyses, we conclude that the increased reactivity of the surface is because B atoms (as a doping species) are more susceptible to nucleophilic attack, and in 2CBN, the $\mathrm{C}-\mathrm{C}$ double bond is more susceptible to alkene addition-like reactions. These changes in the local electronic structure favour particular adsorption configurations. The $\mathrm{OH}$ component bonds strongly to the doping $\mathrm{B}$ atom, whilst $\mathrm{H}$ atoms bond preferentially to $\mathrm{C}$ compared to either $\mathrm{B}$ or $\mathrm{N}$ atoms. Hence, methanol behaves very similarly to water as a polar molecule, because of the $\mathrm{OH}$ group. In the same vein, $\mathrm{H}_{2}$ and methane follow the same trend across the different surfaces, with both binding preferentially on $2 \mathrm{CBN}$, where there is a high energy $\mathrm{C}-\mathrm{C}$ double bond.

The results presented in this study also suggest that adsorption is exothermic in the presence of adsorbed $\mathrm{H}$ atoms (or protons) on the surface. Thus, there could be important implications for the transport properties and chemical reactions of water and other molecules across doped graphene and h-BN membranes, and conditions (acidic or basic) are likely to be useful gauges for altering the interaction with molecules.

Finally, we observe variations in the thermodynamics for the set of molecules considered depending on the surface. Again, we caution that the calculation of reaction barriers and even rates is an important next step, but these results suggest that one can vary the preference for $\mathrm{H}_{2}$ dissociative adsorption to that of water or methanol for example, and consequently alter the course of reaction pathways in either $\mathrm{H}_{2}$ or methanol formation processes. Consider, for example, the wasteful dehydration and methanation reactions in reaction pathways of Cortright et al. catalysed by a metal for $\mathrm{H}_{2}$ production; ${ }^{48}$ wherein $\mathrm{H}_{2}$ is consumed by reacting with $\mathrm{CO}_{2}$ at low temperatures to produce alkanes and water. This reaction can be avoided if methanol, methane, and water are split more readily than $\mathrm{H}_{2}$. According to our findings this might be achievable for methanol and water by doping graphene with BN. Overall, our results indicate that isoelectronically doped graphene and h-BN could exhibit interesting chemical and catalytic activities which could potentially be exploited.

\section{ACKNOWLEDGMENTS}

We are grateful for support from the University College London and Argonne National Laboratory (ANL) through the Thomas Young Centre-ANL initiative. Some of the research leading to these results has received funding from the European Research Council under the European Union's Seventh Framework Programme (No. FP/2007-2013)/ERC Grant Agreement No. 616121 (HeteroIce project). A.M. is supported by the Royal Society through a Wolfson Research Merit Award. O.A.v.L. acknowledges funding from the Swiss National Science foundation (No. PP00P2 138932). In addition, we are grateful for computing resources provided by the London Centre for Nanotechnology and University College London. We would also like to thank Michail Stamatakis for his very helpful insights and suggestions.

${ }^{1}$ E. N. Wang and R. Karnik, Nat. Nanotechnol. 7, 552 (2012).

${ }^{2}$ W. Lei, D. Portehault, D. Liu, S. Qin, and Y. Chen, Nat. Commun. 4, 1777 (2013).

${ }^{3}$ R. Raccichini, A. Varzi, S. Passerini, and B. Scrosati, Nat. Mater. 14, 271 (2015).

${ }^{4}$ B. B. Sales, M. Saakes, J. W. Post, C. J. N. Buisman, P. M. Biesheuvel, and H. V. M. Hamelers, Environ. Sci. Technol. 44, 5661 (2010).

${ }^{5}$ P. Dhiman, F. Yavari, X. Mi, H. Gullapalli, Y. Shi, P. M. Ajayan, and N. Koratkar, Nano Lett. 11, 3123 (2011).

${ }^{6}$ J. Yin, Z. Zhang, X. Li, J. Zhou, and W. Guo, Nano Lett. 12, 1736 (2012).

${ }^{7}$ J. Yin, X. Li, J. Yu, Z. Zhang, J. Zhou, and W. Guo, Nat. Nanotechnol. 9, 378 (2014).

${ }^{8}$ A. Siria, P. Poncharal, A.-L. Biance, R. Fulcrand, X. Blase, S. T. Purcell, and L. Bocquet, Nature 494, 455 (2013).

${ }^{9}$ X. Li, J. Zhao, and J. Yang, Sci. Rep. 3, 1 (2013).

${ }^{10}$ Y. Sun, Q. Wu, and G. Shi, Energy Environ. Sci. 4, 1113 (2011).

${ }^{11}$ Q. Li, B. Guo, J. Yu, J. Ran, B. Zhang, H. Yan, and J. R. Gong, J. Am. Chem. Soc. 133, 10878 (2011).

${ }^{12}$ V. Fthenakis, Renewable Sustainable Energy Rev. 13, 2746 (2009).

${ }^{13}$ L. Ci, L. Song, C. Jin, D. Jariwala, D. Wu, Y. Li, A. Srivastava, Z. Wang, K. Storr, L. Balicas et al., Nat. Mater. 9, 430 (2010).

${ }^{14}$ Z. Liu, L. Ma, G. Shi, W. Zhou, Y. Gong, S. Lei, X. Yang, J. Zhang, J. Yu, K. P. Hackenberg et al., Nat. Nanotechnol. 8, 119 (2013).

${ }^{15}$ X. Fan, Z. Shen, A. Q. Liu, and J.-L. Kuo, Nanoscale 4, 2157 (2012).

${ }^{16}$ B. Xu, Y. H. Lu, Y. P. Feng, and J. Y. Lin, J. Appl. Phys. 108, 073711 (2010).

${ }^{17}$ P. P. Shinde and V. Kumar, Phys. Rev. B 84, 125401 (2011).

${ }^{18}$ Y. Ding, Y. Wang, and J. Ni, Appl. Phys. Lett. 95, 123105 (2009).

${ }^{19}$ R. Zhao, J. Wang, M. Yang, Z. Liu, and Z. Liu, J. Phys. Chem. C 116, 21098 (2012).

${ }^{20}$ N. Kumar, K. Moses, K. Pramoda, S. N. Shirodkar, A. K. Mishra, U. V. Waghmare, A. Sundaresan, and C. N. R. Rao, J. Mater. Chem. A 1, 5806 (2013).

${ }^{21}$ K. Moses, S. N. Shirodkar, U. V. Waghmare, and C. N. R. Rao, Mater. Res. Express 1, 025603 (2014).

${ }^{22}$ C.-K. Chang, S. Kataria, C.-C. Kuo, A. Ganguly, B.-Y. Wang, J.-Y. Hwang, K.-J. Huang, W.-H. Yang, S.-B. Wang, C.-H. Chuang et al., ACS Nano 7, 1333 (2013).

${ }^{23}$ L. Ferrighi, M. I. Trioni, and C. Di Valentin, J. Phys. Chem. C 119, 6056 (2015).

${ }^{24}$ Y. Zhao, L. Yang, S. Chen, X. Wang, Y. Ma, Q. Wu, Y. Jiang, W. Qian, and Z. Hu, J. Am. Chem. Soc. 135, 1201 (2013).

${ }^{25}$ R. Baierle, T. Schmidt, and A. Fazzio, Solid State Commun. 142, 49 (2007).

${ }^{26}$ S. Kattel, P. Atanassov, and B. Kiefer, J. Mater. Chem. A 2, 10273 (2014).

${ }^{27}$ Y. Li, W. Zhou, H. Wang, L. Xie, Y. Liang, F. Wei, J.-C. Idrobo, S. J. Pennycook, and H. Dai, Nat. Nanotechnol. 7, 394 (2012).

${ }^{28}$ D. Sen, R. Thapa, and K. K. Chattopadhyay, ChemPhysChem 15, 2542 (2014).

${ }^{29}$ S. Wang, L. Zhang, Z. Xia, A. Roy, D. W. Chang, J.-B. Baek, and L. Dai, Angew. Chem., Int. Ed. 51, 4209 (2012).

${ }^{30}$ S. Zhong, L. Zhou, L. Wu, L. Tang, Q. He, and J. Ahmed, J. Power Sources 272, 344 (2014).

${ }^{31}$ S. Sinthika, E. M. Kumar, and R. Thapa, J. Mater. Chem. A 2, 12812 (2014).

${ }^{32}$ S. Wang, E. Iyyamperumal, A. Roy, Y. Xue, D. Yu, and L. Dai, Angew. Chem., Int. Ed. 50, 11756 (2011). 
${ }^{33}$ H. Fei, R. Ye, G. Ye, Y. Gong, Z. Peng, X. Fan, E. L. G. Samuel, P. M. Ajayan, and J. M. Tour, ACS Nano 8, 10837 (2014).

${ }^{34}$ L. Joly, G. Tocci, S. Merabia, and A. Michaelides, J. Phys. Chem. Lett. 7, 1381 (2016).

${ }^{35}$ R. J. Baierle, P. Piquini, T. M. Schmidt, and A. Fazzio, J. Phys. Chem. B 110, 21184 (2006).

${ }^{36}$ M. Pizzochero, O. Leenaerts, B. Partoens, R. Martinazzo, and F. M. Peeters, J. Phys.: Condens. Matter 27, 425502 (2015).

${ }^{37}$ M. Chhetri, S. Maitra, H. Chakraborty, U. V. Waghmare, and C. Rao, Energy Environ. Sci. 9, 95 (2016).

${ }^{38}$ C. Tsai, K. Chan, J. K. Nørskov, and F. Abild-Pedersen, Catal. Sci. Technol. 5, 246 (2015).

${ }^{39}$ Y. H. Li, P. F. Liu, L. F. Pan, H. F. Wang, Z. Z. Yang, L. R. Zheng, P. Hu, H. J. Zhao, L. Gu, and H. G. Yang, Nat. Commun. 6, 8064 (2015).

${ }^{40}$ B. Yang, R. Burch, C. Hardacre, G. Headdock, and P. Hu, ACS Catal. 4, 182 (2013).

${ }^{41}$ R. Subbaraman, D. Tripkovic, K.-C. Chang, D. Strmcnik, A. P. Paulikas, P. Hirunsit, M. Chan, J. Greeley, V. Stamenkovic, and N. M. Markovic, Nat. Mater. 11, 550 (2012).

${ }^{42}$ A. U. Nilekar, K. Sasaki, C. A. Farberow, R. R. Adzic, and M. Mavrikakis, J. Am. Chem. Soc. 133, 18574 (2011).

${ }^{43}$ L. C. Grabow and M. Mavrikakis, ACS Catal. 1, 365 (2011).

${ }^{44}$ A. Vojvodic and J. K. Nørskov, Science 334, 1355 (2011).

${ }^{45}$ C. Tsai, K. Chan, J. K. Nørskov, and F. Abild-Pedersen, J. Phys. Chem. Lett. 5, 3884 (2014).

${ }^{46}$ J. K. Nørskov, T. Bligaard, A. Logadottir, S. Bahn, L. B. Hansen, M. Bollinger, H. Bengaard, B. Hammer, Z. Sljivancanin, M. Mavrikakis et al., J. Catal. 209, 275 (2002).

${ }^{47}$ A. Michaelides, Z.-P. Liu, C. Zhang, A. Alavi, D. A. King, and P. Hu, J. Am. Chem. Soc. 125, 3704 (2003).

${ }^{48}$ R. Cortright, R. Davda, and J. A. Dumesic, Nature 418, 964 (2002).

${ }^{49}$ X. Guo, G. Fang, G. Li, H. Ma, H. Fan, L. Yu, C. Ma, X. Wu, D. Deng, M. Wei et al., Science 344, 616 (2014).

${ }^{50}$ G. Kresse and J. Hafner, Phys. Rev. B 47, 558 (1993).

${ }^{51}$ G. Kresse and J. Hafner, Phys. Rev. B 49, 14251 (1994).

${ }^{52}$ G. Kresse and J. Furthmüller, Comput. Mater. Sci. 6, 15 (1996).

${ }^{53}$ G. Kresse and J. Furthmüller, Phys. Rev. B 54, 11169 (1996).

${ }^{54}$ P. E. Blöchl, Phys. Rev. B 50, 17953 (1994).

${ }^{55}$ G. Kresse and D. Joubert, Phys. Rev. B 59, 1758 (1999).

${ }^{56}$ For methanol the separation distance in the z-direction was increased to $20 \AA$ to allow space for the larger adsorbed fragments.

${ }^{57}$ J. P. Perdew, K. Burke, and M. Ernzerhof, Phys. Rev. Lett. 77, 3865 (1996).

${ }^{58} \mathrm{We}$ verified the stability of the doped substrates by calculating their cohesive energies and we find good agreement with other work for similar arrangements of doping atoms ${ }^{17}$. Cohesive energies for the different substrates have been calculated as $E_{c o h}=E_{\text {sheet }}^{\text {tot }}-N_{C} E_{C}^{\text {tot }}-N_{B} E_{B}^{\text {tot }}$ $-N_{N} E_{N}^{\text {tot }}$ where $E_{\text {sheet }}^{\text {tot }}, E_{C}^{\text {tot }}, E_{B}^{\text {tot }}$ and $E_{N}^{\text {tot }}$ are the total energies of the sheet and gaseous $\mathrm{C}, \mathrm{B}$ and $\mathrm{N}$ atoms in the unit cell, respectively, and $N_{C}, N_{B}$ and $N_{N}$ are the numbers of $\mathrm{C}, \mathrm{B}$ and $\mathrm{N}$ atoms in the unit cell. The doped sheets in this study have cohesive energies between that of graphene and h-BN, and the four substrates range between -7.06 and $-7.84 \mathrm{eV} /$ atom.

${ }^{59}$ S. Hu, M. Lozada-Hidalgo, F. Wang, A. Mishchenko, F. Schedin, R. Nair, E. Hill, D. Boukhvalov, M. Katsnelson, R. Dryfe et al., Nature 516, 227 (2014).
${ }^{60}$ P. Cabrera-Sanfelix and G. R. Darling, J. Phys. Chem. C 111, 18258 (2007).

${ }^{61}$ S. Xu, S. Irle, D. Musaev, and M. Lin, J. Phys. Chem. A 109, 9563 (2005).

${ }^{62}$ Y. Lin, F. Ding, and B. I. Yakobson, Phys. Rev. B 78, 041402 (2008).

${ }^{63}$ D. Boukhvalov, M. Katsnelson, and A. Lichtenstein, Phys. Rev. B 77, 035427 (2008).

${ }^{64}$ R. Balog, B. Jørgensen, L. Nilsson, M. Andersen, E. Rienks, M. Bianchi, M. Fanetti, E. Lægsgaard, A. Baraldi, S. Lizzit et al., Nat. Mater. 9, 315 (2010).

${ }^{65}$ Ž. Šljivančanin, M. Andersen, L. Hornekær, and B. Hammer, Phys. Rev. B 83, 205426 (2011).

${ }^{66}$ P. Merino, M. Švec, J. I. Martnez, P. Mutombo, C. Gonzalez, J. A. MartnGago, P. L. de Andres, and P. Jelinek, Langmuir 31, 233 (2015).

${ }^{67} \mathrm{The} 0.04 \mathrm{eV}$ difference between cis-para and trans-ortho adsorption configurations remained the same using a denser $6 \times 6 \times 1 \mathrm{k}$-point mesh.

${ }^{68}$ Y. Ding, M. Iannuzzi, and J. Hutter, J. Phys. Chem. C 115, 13685 (2011).

${ }^{69}$ B. Wang and M.-L. Bocquet, J. Phys. Chem. Lett. 2, 2341 (2011).

${ }^{70}$ B. Wang, S. Günther, J. Wintterlin, and M.-L. Bocquet, New J. Phys. 12, 043041 (2010).

${ }^{71}$ J. Wintterlin and M.-L. Bocquet, Surf. Sci. 603, 1841 (2009).

${ }^{72}$ J. G. Díaz, Y. Ding, R. Koitz, A. P. Seitsonen, M. Iannuzzi, and J. Hutter, Theor. Chem. Acc. 132, 1350 (2013).

${ }^{73}$ S. Altenburg, J. Kröger, B. Wang, M.-L. Bocquet, N. Lorente, and R. Berndt, Phys. Rev. Lett. 105, 236101 (2010).

${ }^{74}$ M. Lattelais and M.-L. Bocquet, J. Phys. Chem. C 119, 9234 (2015).

${ }^{75} \mathrm{X}$. Li, J. Feng, E. Wang, S. Meng, J. Klimeš, A. Michaelides et al., Phys. Rev. B 85, 085425 (2012).

${ }^{76}$ S. Joshi, D. Ecija, R. Koitz, M. Iannuzzi, A. P. Seitsonen, J. Hutter, H. Sachdev, S. Vijayaraghavan, F. Bischoff, K. Seufert et al., Nano Lett. 12, 5821 (2012).

${ }^{77}$ R. F. Bader, Atoms in Molecules (Wiley Online Library, 1990).

${ }^{78}$ G. Henkelman, A. Arnaldsson, and H. Jónsson, Comput. Mater. Sci. 36, 354 (2006).

${ }^{79}$ Of course there are many ways to project charges onto atoms and Bader charges discussed here are simply used for pinpointing the relevant trends in the materials.

${ }^{80}$ L. Schimka, J. Harl, A. Stroppa, A. Grüneis, M. Marsman, F. Mittendorfer, and G. Kresse, Nat. Mater. 9, 741 (2010).

${ }^{81}$ J. Klimeš and A. Michaelides, J. Chem. Phys. 137, 120901 (2012).

${ }^{82}$ Y. S. Al-Hamdani, M. Ma, D. Alfè, O. A. von Lilienfeld, and A. Michaelides, J. Chem. Phys. 142, 181101 (2015).

${ }^{83}$ Y. S. Al-Hamdani, D. Alfè, O. A. von Lilienfeld, and A. Michaelides, J. Chem. Phys. 141, 18C530 (2014).

${ }^{84}$ M. Dion, H. Rydberg, E. Schröder, D. C. Langreth, and B. I. Lundqvist, Phys. Rev. Lett. 92, 246401 (2004).

${ }^{85}$ A. D. Becke, J. Chem. Phys. 84, 4524 (1986).

${ }^{86}$ J. Klimeš, D. R. Bowler, and A. Michaelides, Phys. Rev. B 83, 195131 (2011).

${ }^{87}$ A. D. Becke, J. Chem. Phys. 98, 5648 (1993).

${ }^{88}$ C. Lee, W. Yang, and R. G. Parr, Phys. Rev. B 37, 785 (1988).

${ }^{89}$ S. H. Vosko, L. Wilk, and M. Nusair, Can. J. Phys. 58, 1200 (1980).

${ }^{90}$ P. J. Stephens, F. J. Devlin, C. F. Chabalowski, and M. J. Frisch, J. Phys. Chem. 98, 11623 (1994). 